\title{
La encrucijada de la integración regional y el nuevo regionalismo
}

\author{
René Hernández \\ Comisión Económica para América Latina y el Caribe
}

\section{Resumen}

El artículo presenta una reseña del panorama actual de la integración regional y el nuevo regionalismo en América Latina y el Caribe resaltando algunos de los temas emergentes como la salida del Reino Unido de la Unión Europea (Brexit), las negociaciones megarregionales, la renegociación del Tratado de Libre Comercio de América del Norte (TLCAN), el Acuerdo de Asociación Transpacífico (TPP) y la Alianza del Pacífico (AP). Se plantean además los desafíos de los procesos de integración regional y nuevo regionalismo en áreas que van más allá del intercambio comercial y que suponen la gradual coordinación macroeconómica y en diversas políticas y normas incluyendo infraestructura, energía y ámbitos regulatorios. Se destaca el desafío de fortalecer el vínculo con China y Asia-Pacífico, el comercio de servicios, la coordinación regulatoria y la integración productiva como mecanismos de una integración regional más profunda.

Palabras clave: integración regional, regionalismo, nuevo regionalismo, acuerdos comerciales..

The crossroad of regional integration and the new regionalism

\begin{abstract}
The article presents a review of the current panorama of regional integration and the new regionalism in Latin America and the Caribbean highlighting some of the emerging issues such as the exit of the United Kingdom from the European Union (Brexit), the megaregional negotiations, the renegotiation of the Treaty of Free Trade of North America (NAFTA), the Trans-Pacific Partnership Agreement (TPP) and the Pacific Alliance (AP). The challenges of the processes of regional integration and new regionalism in areas beyond trade exchange which involve the gradual macroeconomic coordination and also in policies and norms including infrastructure, energy and regulatory areas are also considered. The challenge of strengthening the link with China and Asia-Pacific, trade in services, regulatory coordination and productive integration as mechanisms of deeper regional integration stands out.
\end{abstract}

Keywords: regional integration, regionalism, new regionalism, trade agreements..

*Dirección de correspondencia [Correspondence

address]: René Hernández, Comisión Económica para

América Latina y el Caribe

E-mail: rene.hernandez64@gmail.com 


\section{Introducción}

La dinámica del regionalismo y la integración regional en América Latina y el Caribe han sufrido importantes transformaciones en las últimas dos décadas. Varios factores explican estos cambios. Por una parte, el malestar y descontento social suscitado en varios países de la región con los resultados de las reformas neoliberales de los años noventa implementadas bajo los preceptos del Consenso de Washington, permitió la llegada al poder de alianzas y coaliciones políticas de tendencia neoliberal y conservadora, las cuales se trasladaron al plano de la integración. Como es de esperarse y con diversos matices y énfasis, varios de los gobiernos que asumieron el poder en la región en los últimos años comparten una postura crítica o al menos de escepticismo sobre los procesos de apertura comercial y de libre comercio llevados a cabo a partir de los años noventa, así como sobre el énfasis en la dimensión comercial que habría caracterizado a los proyectos de integración durante los años noventa. En algunos casos, estos gobiernos han procurado ampliar la agenda de la integración hacia otras áreas, tales como las dimensiones política y social en sus distintas formulaciones y vertientes.

El artículo parte de un marco analítico que recurre a algunos elementos de la economía política internacional y del institucionalismo histórico, para explicar cómo la integración y el regionalismo latinoamericano responden a factores endógenos y exógenos y al marco de políticas de ajuste imperante. Este marco analítico, no será tratado con detalle en este artículo ya que se persigue el objetivoespecifico: presentar los desafíos de la integración regional y el nuevo regionalismo en América Latina y el Caribe (ALC) y proponer de manera estilizada algunas ideas para renovar el debate sobre la integración regional y el nuevo regionalismo (Sanahuja, 2016).

Se plantea la hipótesis que con una agenda comercial proteccionista y unilateralista como la de la Administración Trump, América Latina está obligada a replantearse sus opciones de inserción internacional y de integración regional, teniendo en consideración la salida del Reino Unido de la Unión Europea (Brexit), las negociaciones megarregionales, la renegociación del Tratado de Libre Comercio de América del Norte (TLCAN), la Alianza del Pacífico (AP) y la negativa del Gobierno de Estados Unidos de proseguir con las negociaciones y firma del Acuerdo de Asociación Transpacífico (TPP). En un contexto de creciente proteccionismo, la integración regional y el nuevo regionalismo pueden constituirse en los pilares que impulsen y sostengan un desarrollo más inclusivo para toda la región.
Además de la introducción en la primera parte se presenta una síntesis con las ideas centrales del regionalismo liberal y del nuevo regionalismo. En la segunda parte se hace una reseña de los temas emergentes y del debate reciente, como el Brexit, las negociaciones megarregionales, la renegociación del TLCAN, la Alianza del Pacífico, entre otros. La tercera parte expone algunas ideas orientadas a renovar el nuevo regionalismo y la integración regional en $\mathrm{ALC}^{1}$. Se concluye con una presentación de los principales desafíos que enfrenta la región en materia de integración regional y nuevo regionalismo.

\section{El regionalismo liberal y el re- gionalismo desarrollista}

Los actuales procesos de regionalización e integración regional se enmarcan dentro de un fenómeno más amplio que diversos autores definen como el "nuevo regionalismo" a escala global (Hettne, 1999; Söderbaum y Shaw, 2003).

El análisis del "viejo regionalismo" que se identifica con el regionalismo liberal, se remonta a la teoría clásica del comercio internacional y a la versión neoclásica de Heckscher-Ohlin-Samuelson. Dentro de esta visión neoclásica de la teoría del comercio internacional, destaca la obra de Viner $(1999,1954)$ con los conceptos de creación y desviación de comercio.

Esta teoría tradicional y de "regionalismo liberal" contrasta no solamente con el "nuevo regionalismo" (Robson, 1993; Ethier, 1998), sino además con el "regionalismo desarrollista" propugnado por algunos teóricos relacionados con los países en desarrollo, especialmente de África, o con el regionalismo "abierto" impulsado por la Comisión Económica de América Latina y el Caribe (CEPAL) desde mediados de los años noventa, principalmente en América Latina y el Caribe (Comisión Económica para América Latina y el Caribe (CEPAL), 1994; Alves Teixeira y Desiderá Neto, 2012).

Prima facie, el regionalismo "abierto" se opone al regionalismo "cerrado". El primero está fundamentado en la apertura comercial y en los principios y disciplinas del sistema multilateral de comercio. El segundo en cambio, aboga por una agenda proteccionista y unilateralista.

En América Latina, regionalismo abierto y nue-

\footnotetext{
${ }^{1}$ Integración regional y regionalismo no son formalmente equivalentes y la literatura distingue las diferencias. En otros casos, la distinción es difusa y ambigua. Véase una síntesis de esta discusión en Malamud (2013).
} 
vo regionalismo son empleados indistintamente para denotar el proceso caracterizado por la liberalización del comercio de bienes y servicios, y de los movimientos de capital.

La CEPAL contribuyó al debate sobre los procesos de integración regional con una propuesta de "regionalismo abierto" que combinaba, los acuerdos subregionales de liberalización con la apertura unilateral y el avance hacia la integración hemisférica.

Comisión Económica para América Latina y el Caribe (CEPAL) (1994) define el regionalismo abierto como: "...el proceso de creciente interdependencia económica a nivel regional, impulsado tanto por acuerdos preferenciales de integración como por otras políticas en un contexto de creciente apertura y desreglamentación, con el objeto de aumentar la competitividad de los países de la región y de constituir, en la medida de lo posible, un cimiento para una economía internacional más abierta y transparente. Con todo, de no producirse ese escenario óptimo, el regionalismo abierto de todas maneras cumpliría una función importante, en este caso un mecanismo de defensa de los efectos de eventuales presiones proteccionistas en mercados extraregionales."

Dos temas surgen con preeminencia en el debate actual: Una tendencia hacia mayores iniciativas de integración del comercio de servicios y de integración regulatoria.

La literatura reciente discute los objetivos económicos y políticos perseguidos por la integración regional (y sus efectos, tanto los buscados como los que surgen sin haber sido buscados). Desde una perspectiva estrictamente económica, la integración es analíticamente abordada sobre todo como un instrumento de desarrollo (o de competitividad y crecimiento). Este fin último es perseguido a través de una serie de objetivos intermedios, normalmente analizados desde dos puntos de vista. En primer lugar, aumenta la competencia y se generan economías de escala como resultado de la ampliación del mercado y del contacto más estrecho entre productores de los distintos Estados miembros. En segundo lugar, la integración regional cambia los patrones de los flujos de comercio, así como la localización de la producción. Se requiere una perspectiva dinámica para dimensionar estas ventajas porque los beneficios netos de la dinámica de integración, combinados con los llamados efectos notradicionales, tales como el efecto de señalamiento y de "cerrojo" o "consolidación" (lock-in), pueden ser mucho mayores que sus efectos estáticos de reasignación.

Ahora bien, el crecimiento de los bloques comer- ciales regionales ha constituido uno de los principales avances en el campo de las relaciones internacionales en años recientes. En la actualidad, prácticamente todos los países pertenecen a por lo menos uno de estos bloques. Además de su incremento en cantidad, durante los últimos veinte años se han observado también cambios cualitativos en los acuerdos regionales de integración. Hay tres de éstos que merecen especial atención:

1. El paso de un modelo de regionalismo cerrado a uno más abierto. La nueva ola de acuerdos regionales de integración (que comprende la restauración de algunos convenios antiguos) por lo general revela una postura de apertura hacia el exterior y un compromiso más dado a fomentar el comercio internacional que a controlarlo.

2. El reconocimiento de que una integración efectiva o de facto exige, además de la reducción de aranceles y cuotas, la eliminación de otras barreras al comercio, lo que se ha denominado "integración profunda" 2 .

3. El advenimiento de los bloques comerciales en los que los países de altos ingresos y los países en desarrollo actúan como socios en pie de igualdad -conocidos como acuerdos Norte-Sur.

Son muchos los factores que ha servido de base para el reciente surgimiento del regionalismo. Para Schiff y Winters (2003) algunos de los más importantes son:

1. El deseo de los gobiernos de comprometerse con mejores políticas -incluida la propia democracia- y con hacer conocer dichos compromisos a los inversionistas nacionales y extranjeros.

\footnotetext{
${ }^{2}$ La tesis de que existen "cinco fases" sucesivas en la integración (zona de libre comercio, unión aduanera, mercado común, unión monetaria y unión política) es rigurosamente falsa. Basta contrastarla con el North American Free Trade Agreement (NAFTA) o Tratado de Libre Comercio de América del Norte (TLCAN) y el propio Mercado Común del Sur (MERCOSUR). El primero incluye aspectos muy importantes de mercado común en materias que van más allá del comercio de bienes (servicios, inversiones), y en cualquier caso mucho más importantes y amplios que los que incluye el MERCOSUR, sin haberse ni tan sólo planteado el "paso" por la fase precedente, de unión aduanera. Y el MERCOSUR ha tocado muchos temas propios de la unión política (desde la cláusula democrática a la cooperación educativa, judicial y policial) sin haber avanzado prácticamente nada en materia de mercado común. Tampoco el proceso europeo se ajusta a aquella sucesión de fases porque muchos aspectos relativos al mercado común se plantearon en el momento fundacional y no en un momento ulterior. Y, por último, ASEAN demuestra que se puede invertir el proceso y comenzarse con la política para acabar en el comercio de bienes.
} 
2. El anhelo de obtener un acceso más seguro a los mercados más importantes.

3. Las presiones de la globalización y de la hiperglobalización, que obligan a empresas y países a buscar eficiencias entrando en contacto con mercados de tamaño superior, mayor competencia y acceso a tecnologías e inversiones extranjeras.

4. El deseo de los gobiernos de mantener su soberanía, conjugándola con la de otros países, en las áreas de gestión económica en las que la mayoría de las naciones-estado son demasiado pequeñas para actuar individualmente por su cuenta.

5. El anhelo de impulsar el sistema multilateral hacia una acción más rápida y profunda en áreas determinadas, para demostrar que el Acuerdo General sobre Aranceles Aduaneros y Comercio (GATT) no era el único recurso y crear bloques más poderosos que funcionaran en el marco del mismo GATT.

6. El deseo de ayudarles a los países vecinos a estabilizarse y progresar, tanto por razones altruistas como para atenuar efectos indirectos de malestar social en la población generados por la hiperglobalización ${ }^{3}$.

7. El temor de quedarse por fuera del resto del mundo cuando éste sea absorbido por el regionalismo, la hiperglobalización o por la dinámica de las cadenas globales de valor y las redes globales de producción, bien sea porque esto es en realidad perjudicial o simplemente porque "si todos los demás lo están haciendo, ¿no lo deberíamos hacer también nosotros?".

Entre las causas más fundamentales, se destacarían la caída de la hegemonía soviética, que indujo a los países de Europa Oriental y el Báltico a acoger la democracia y el capitalismo, y a los de Europa Occidental a buscar formas de consolidar y acelerar su transición a los mismos; el cambio en la forma de entender el papel que juega la apertura en el desarrollo, junto con el anhelo político natural de restringir los temidos costos del ajuste de la apertura unilateral no discriminatoria; la necesidad de

\footnotetext{
${ }^{3}$ Se denomina hiperglobalización al creciente flujo transfronterizo de bienes, servicios y capitales. El malestar contra la hiperglobalización se evidencia por ejemplo por los resultados del referéndum de junio de 2016 sobre la salida del Reino Unido de la Unión Europea (Brexit) o por los resultados de las elecciones presidenciales de los Estados Unidos celebradas en noviembre de 2016, junto con un creciente número de movimientos antiglobalización en los que se cuestionan los beneficios del libre comercio y la inversión de empresas transnacionales (Comisión Económica para América Latina y el Caribe (CEPAL), 2016).
}

crear una dinámica en el orden nacional para las reformas requeridas para lograr una mayor apertura y, al mismo tiempo, reducir a un mínimo los problemas políticos que puedan perturbar las fuentes de ingresos y rentas existentes; y el cambio reciente de actitud de Estados Unidos y el Presidente Trump frente a los bloques comerciales. En el pasado, los vaivenes en la actitud de Estados Unidos y de administraciones previas a la del Presidente Trump (de una postura muy entusiasta a una hostilidad activa), no sólo fomentaron los acuerdos regionales de integración, sino que disminuyeron la presión diplomática -tanto manifiesta (por medio del GATT) como tácita- que desanimaba a los países a formar dichos acuerdos. Esto se originó, al menos en parte, en la frustración expresada ante la lentitud del proceso multilateral.

Igualmente importantes, aunque menos divulgados, fueron la creciente influencia de los grupos de lobby empresariales de Estados Unidos en la formulación de políticas, la decreciente competitividad de la industria estadounidense y la escasa voluntad de asumir los costos que implica la gestión del sistema global, sin recibir beneficios directos en forma de acceso a mercados. A raíz de la terminación de la Guerra Fría desapareció la justificación política preponderante de responder por esos costos y el debate se centró más directamente en los objetivos mercantilistas, como siempre había sido el caso en la mayoría de los países pequeños.

\section{Negociaciones megarregionales}

A partir de la década de los noventa se entró a una nueva fase de las relaciones económicas internacionales, caracterizada por un crecimiento acelerado de los flujos transfronterizos de bienes, servicios y capitales, dando origen a un proceso irreversible de hiperglobalización y conformación de bloques económicos de escala global y negociaciones megarregionales. El más sobresaliente y controvertido a la vez, es el Acuerdo de Asociación Transpacífico (TPP).

La hiperglobalización en la región latinoamericana se ha desarrollado en un contexto de reducción de la pobreza y aumento de la desigualdad del ingreso, generando como consecuencia fuertes tensiones sociales y políticas recientes que emergen producto de la contradicción entre los objetivos de equilibrio externo de los países y la dinámica propia de la hiperglobalización.

América Latina está compuesta por economías muy heterogéneas, con capacidades tecnológicas diferenciadas por sector y rama de actividad económi- 
ca y esto propicia la generación de una tendencia secular de desequilibrios en cuenta corriente en estos países. Cuando los desbalances regionales y globales persisten, los países deficitarios no tienen más alternativa que contraer su ritmo de crecimiento, sus niveles de empleo y el nivel de gasto social para evitar mayores desequilibrios externos. En los países desarrollados, se impacta el nivel de empleos y salarios y en general, todo ello exacerba el descontento por la hiperglobalización (Comisión Económica para América Latina y el Caribe (CEPAL), 2016).

Una de las manifestaciones del descontento por la hiperglobalización por parte de los países desarrollados, es el resultado del referéndum a favor de la salida del Reino Unido de la Unión Europea, realizado en junio de 2016, el cual tuvo como efecto inmediato el aumento de los niveles de incertidumbre en los mercados de capitales y colateralmente exacerbó el sesgo recesivo que se traía de la economía mundial. Asimismo, en 2017 se inició una ronda de negociaciones para definir los términos de la nueva relación entre el Reino Unido y la Unión Europea. Quizás el tema clave sea la definición del acceso libre de ese país al Mercado Único Europeo y la posibilidad de que el Reino Unido mantenga la libertad de establecimiento en su territorio para los ciudadanos de cualquier país miembro de la Unión Europea. No debe causar extrañeza ni sorpresa que los temas de mayor control migratorio hayan estado en la campaña a favor del brexit. Persiste sin embargo, la duda sobre la postura que adoptará la política comercial británica frente a su futura participación en acuerdos comerciales suscritos con terceros países, o frente a sus negociaciones comerciales en curso (en particular, con los Estados Unidos en el marco de la Asociación Transatlántica de Comercio e Inversión (TTIP).

Por otra parte, otra de las manifestaciones del descontento por la hiperglobalización fue una de las promesas de campaña del Presidente Trump y se relacionaba con la renegociación del Tratado de Libre Comercio de América del Norte (TLCAN) con el fin de devolver a los Estados Unidos los empleos deslocalizados en México y convencer a las empresas norteamericanas a que realicen sus nuevas inversiones al norte de la frontera entre Estados Unidos y México.

Es de esta manera, que los gobiernos estadounidense, mexicano y canadiense buscan actualizar un tratado con casi veinticinco años de vigencia que fue firmado en 1992 y que entró en vigor en 1994. El TLCAN propició de facto una de las mayores zonas de libre comercio en el mundo y la frontera sur de Estados Unidos y México es transitada cada día por cerca de un millón de personas y casi medio millón de vehículos.
Las negociaciones iniciaron recientemente y lo que está en juego no es para nada despreciable. De acuerdo a datos de la CEPAL, Canadá y México son el segundo y tercer socio comercial de Estados Unidos, siendo China el primer socio comercial. Para Canadá, Estados Unidos y México son el primer y tercer socio comercial, respectivamente y para México, Estados Unidos y Canadá son sus dos primeros socios comerciales, medido por volumen de comercio.

Sin duda, México llega a la mesa de negociaciones en desventaja relativa, ya que su economía presenta estructuralmente una diversificación exportadora mucho menor a pesar de ser una economía muy abierta $^{4}$, ya que más del $86 \%$ de sus exportaciones no petroleras tiene como principal destino a los Estados Unidos y solamente el 4,5\% de las exportaciones mexicanas van a los 28 países de la Unión Europea; el $1,2 \%$ se destinan a China y el $0,7 \%$ a Brasil, la primera y más grande economía de la región.

El déficit comercial, las normas de origen y los asuntos laborales parecieran ser los tres temas que marcarán la pauta de las negociaciones. Es probable que los negociadores estadounidenses busquen mecanismos para disminuir el déficit comercial con México y Canadá y esto se convierta en uno de los temas más complejos a tratar, por los desbalances globales existentes y por los ajustes requeridos en los niveles de empleo para mantener los equilibrios externos.

En el caso de que las negociaciones lleven a una eventual ruptura del TLCAN, se puede prever que el intercambio comercial pasaría a regirse por la normativa de la Organización Mundial de Comercio (OMC), lo cual supondría en la práctica la imposición de un arancel medio de 3,5\% a los productos estadounidenses importados por México con consecuencias económicas de gran magnitud para sectores estratégicos del acuerdo como el automotriz y electrónico.

Por otra parte, y como otra de las manifestaciones de descontento por la hiperglobalización se encuentra el anuncio del Presidente Trump de retirarse del Acuerdo de Asociación Transpacífico (TPP). El 4 de febrero de 2016, 12 países de América Latina y el Caribe, América del Norte, Asia y Oceanía suscribieron el Acuerdo de Asociación Transpacífico (TPP), después de casi seis años de negociaciones. El TPP es el primer acuerdo de un conjunto nuevo de negociaciones comerciales de gran alcance,

\footnotetext{
${ }^{4}$ México cuenta con una docena de tratados comerciales firmados con 46 países a lo largo y ancho del mundo. Sin embargo, su dependencia de Estados Unidos sigue siendo casi absoluta.
} 
conocidas como megarregionales. El TPP establecería la mayor área de libre comercio del mundo, y la segunda más grande, después de la Unión Europea, medida por el monto del comercio entre ellos. En conjunto, sus miembros representan el $38 \%$ del PIB mundial y un cuarto del comercio mundial. De igual manera, en 2015 recibieron un tercio de los flujos mundiales de IED y fueron el origen de un $40 \%$ de esos flujos (Comisión Económica para América Latina y el Caribe (CEPAL), 2016).

El TPP a diferencia de la mayoría de los acuerdos comerciales existentes, se caracteriza por su naturaleza plurilateral e interregional y por su amplia cobertura temática. Incluye los temas tradicionales de acceso a los mercados de bienes, servicios, inversión y contratación pública, establece reglas sobre temas que no han sido normados por los acuerdos de la OMC como el del comercio electrónico, las empresas estatales, la coherencia regulatoria y diversos aspectos del mercado de trabajo y medioambientales. En áreas como la propiedad intelectual, se esperaría que el TPP impulse o propicie un proceso progresivo de armonización regulatoria entre sus miembros, pese a las asimetrías de desarrollo institucional y de los marcos legales y normativos prevalecientes en los países miembros (Comisión Económica para América Latina y el Caribe (CEPAL), 2016).

El TPP es un acuerdo de una magnitud, alcance y complejidad únicos y sin precedentes. Ha sido tan controvertido que el presidente Trump lo utilizó en su campaña presidencial para oponerse a los tratados comerciales suscritos por administraciones previas y a revisar los que estaban en proceso de negociación. En este sentido, existe una fuerte incertidumbre sobre el futuro del acuerdo ya que para que entre en vigor debe ser ratificado como mínimo por seis de sus miembros que en conjunto representen al menos el $85 \%$ del PIB total de los países firmantes, lo cual implica que en la práctica, su entrada en vigor es imposible sin la ratificación de los Estados Unidos y Japón (Comisión Económica para América Latina y el Caribe (CEPAL), 2016).

Existe además más incertidumbre sobre el posible impacto del TPP en países como Chile, México y Perú y sobre la forma en que una eventual suscripción del tratado conllevaría o propiciaría en cierta medida, procesos auténticos de diversificación y sofisticación de la estructura productiva y de la base exportadora de estos países.

La salida de los Estados Unidos del TPP ha impulsado a Japón a asumir el liderazgo y mantener vivo el acuerdo. Ahora, el TPP ha resurgido como el Tratado Integral y Progresista de Asociación Transpacífico (CPTPP, por sus siglas en inglés, Comprehensive and Progressive Agreement for Trans-

\section{Pacific Partnership).}

El 8 de marzo de 2018, en Santiago de Chile, los ministros de comercio de los 11 países del CPTPP firmaron dicho acuerdo que entrará en vigor 60 días después de que al menos seis de los signatarios notifiquen por escrito la ratificación del Tratado a Nueva Zelandia, país depositario del CPTPP. De esta manera, se mantiene el TPP fundamentalmente sin cambios y se suspenden 20 medidas que, al haber sido prioritarias para los Estados Unidos, pero problemáticas para la mayoría de sus miembros, permiten que el resto de los países lo puedan aprobar en un futuro cercano (De la Mora Sánchez, 2018).

Por último, La Alianza del Pacífico (AP) se está posicionado en la vanguardia de las negociaciones comerciales internacionales, atrayendo a los países asociados e incorporando las disciplinas del TPP. La AP podría constituirse en una plataforma de atracción de nuevas inversiones y nuevas oportunidades de comercio para sus adherentes. Por otra parte, las negociaciones entre bloques comerciales, para el caso entre la AP y el MERCOSUR abre nuevos espacios para explotar economías de escala, economías de localización y aglomeración. Esto permitiría el escalamiento al interior de las principales cadenas globales de valor (CGV) y redes globales de producción (RGP) (De la Mora Sánchez, 2018).

\section{La renovación del nuevo regio- nalismo y de la integración re- gional $^{5}$}

En el pasado desde fines de la Segunda Guerra Mundial hasta principios de la década de los 70, la integración económica de ALC formó parte de un modelo de industrialización dirigida por el Estado promovido por la CEPAL y conocido ampliamente como estrategia de industrialización por sustitución de importaciones (ISI), privilegiando los instrumentos dirigidos a incrementar la producción que satisficiera la demanda regional (arancel externo común, incentivos fiscales, mecanismos de pago, libre comercio de bienes industriales, arreglos institucionales) $)^{6}$.

\footnotetext{
${ }^{5}$ Esta sección se basa fundamentalmente en un artículo de Osvaldo Rosales (2008) "Integración regional: propuestas de renovación", presentado en el Seminario Internacional Paradojas de la Integración en América Latina.

${ }^{6}$ Por razones puramente analíticas pero también de exposición, se prefiere utilizar el término "industrialización dirigida por el Estado" en lugar de "industrialización por sustitución de importaciones", ya que siguiendo la narrativa de Cárdenas et al. (2003), la sustitución de importaciones no fue ni el elemento más destacado del modelo a lo largo del tiempo, ni una característica compartida por todos los
} 
En el nuevo regionalismo o regionalismo abierto, el proceso de integración regional puede convertirse en un medio para elevar el grado de inserción de los países en la economía internacional, lo cual exige aprovechar los beneficios potenciales de economías de escala, la reducción de costos de transacción, los incrementos de la eficiencia y la mayor seguridad derivada de políticas que se afianzan por la acción colectiva (Comisión Económica para América Latina y el Caribe (CEPAL), 1994).

La experiencia de América Latina y el Caribe muestra que el comercio intra-regional favorece la diversificación exportadora, es más amigable con las pequeñas y medianas empresas (PYMES) y más intensivo en valor agregado que el dirigido al resto del mundo. Esa es la buena noticia. La mala es que el despegue de la integración regional y subregional es demasiado lento para los tiempos actuales. Los esfuerzos de integración en los últimos 25 años se han dado en un contexto económico poco favorable para la región, tanto en crecimiento como en equidad. No parece justo, por ende, culpar a la integración de un contexto de tres décadas de bajo crecimiento, profundas fragmentaciones sociales y marcadas turbulencias políticas, incluyendo la pérdida y posterior reconquista de la democracia en un número significativo de países de la región. Dado este contexto, mal se podría exigir a la integración avances muy superiores a los que muestra la región en el resto de indicadores económicos y sociales (Rosales, 2008).

Lamentablemente esas tres décadas coinciden con cambios drásticos en la economía mundial. Se intensifica la globalización, se acelera el cambio tecnológico y emergen nuevos y agresivos competidores (China e India, acompañados del resto de Asia Pacífico), todo lo cual está conduciendo a una modificación drástica en el mapa mundial de intercambios, de desaceleración de los flujos y volúmenes de comercio a escala global, de la inversión extranjera directa y otros flujos financieros producto del bajo crecimiento económico mundial después de la crisis de 2007-2008, de ventajas comparativas, de cadenas globales y regionales de valor ${ }^{7}$ y de localización y

países desde fines de la Segunda Guerra Mundial hasta la década de los 70, período en el cual se aplicó este modelo de desarrollo, y que en los hechos, atravesó por diferentes etapas, diversidad en la aplicación de instrumentos y énfasis bastante diferenciados. De hecho, en economías pequeñas de la región, la industrialización no sustituyó por completo al modelo primario-exportador y dio lugar a esquemas mixtos que combinaban la sustitución de importaciones con la promoción de exportaciones (Véase Rosenthal, 1998, 2004).

${ }^{7}$ La participación de América Latina y el Caribe en las cadenas globales de valor se incrementó durante este siglo, pero sigue estando por debajo del promedio mundial y consiste principalmente en el suministro de materias primas para las exportaciones de terceros países. Véase Comisión Económica para América Latina y el Caribe (CEPAL) (2016) relocalización de la producción y de las inversiones.

En 2015, el valor del comercio mundial de bienes se redujo un $14 \%$, mientras su volumen creció un $2,7 \%$. En 2016, el volumen del comercio mundial de bienes crecería un 1,7\%, la menor expansión desde la crisis económica de 2007-2008 y el quinto año consecutivo de crecimiento por debajo del $3 \%$. El comercio mundial de servicios también disminuyó su valor su valor en 2015, aunque fue menos pronunciado que el comercio de bienes $(-6,4 \%$ ) (Comisión Económica para América Latina y el Caribe (CEPAL), 2016).

Es respecto de este contexto global de nuevas amenazas y desafíos que el balance de la integración aparece como insuficiente. Enfrentados a tales desafíos, la brecha entre retórica y práctica integracionista aparece como elevada o desproporcionada ya que las principales decisiones empresariales no se apoyan en los esquemas de integración; la integración no está en el centro de las agendas políticas y cuando parece estarlo, apenas logra trascender el ámbito formal de los anuncios. De esta manera, la actual integración no parece colaborar mucho en los desafíos de competitividad, diversificación exportadora e innovación tecnológica. Con la irrupción de China y otras economías asiáticas en el concierto global, se agrega la serie de acuerdos bilaterales de comercio entre varias economías de la región y Estados Unidos o la Unión Europea, todo lo cual plantea a la región severos desafíos de competitividad y de aggiornamento a sus esquemas de integración (Rosales, 2008).

La tarea de la integración es de por si complicada. La complejidad intrínseca de cualquier proceso integracionista se ve hoy agravada por la confluencia de estos factores: un balance poco satisfactorio, la magnitud de los actuales desafíos, la difusión de acuerdos bilaterales con países extra-zona y la debilidad de liderazgos que promuevan avances prácticos en la integración. En ese cuadro no debiera sorprender una acentuación en la diversidad de opciones respecto de cómo integrarse a la economía mundial.

Hay conocidas y marcadas diferencias estructurales así como otras de política. Entre las primeras, existen diferencias de tamaño, de estructura productiva y exportadora, de ventajas comparativas, de estructura de los principales mercados de destino y de grado de complementación o sustitución con los principales productos agrícolas de las economías industrializadas que mantienen elevados niveles de subsidio a la exportación o de apoyo doméstico a sus productores. Las diferencias de política se relacionan con el rol que cada país aspira a jugar en

y Hernández et al. (2016). 
la economía mundial y regional, el nivel de sus aspiraciones geopolíticas y, por ende, del poder negociador, de la estructura de alianzas, todo lo cual termina reflejándose en la política comercial.

Desde este punto de vista, la difusión de acuerdos bilaterales de tipo Norte-Sur probablemente se explique por una combinación de incertidumbre respecto al ritmo, timing y profundidad de las negociaciones multilaterales así como cierto escepticismo respecto de las posibilidades efectivas de avances en la integración. Vale decir, los Tratados de Libre Comercio (TLC) corresponden en el mejor de los casos, a una tercera mejor opción, después de la liberalización multilateral y de la integración ${ }^{8}$. Una vez que estas dos primeras opciones no responden en los plazos y profundidad en que los gobiernos necesitan las respuestas comerciales y económicas, surgiría entonces el instrumento bilateral.

El desafío es entonces aún más complejo. Se trata de fortalecer los procesos de integración regional, buscando mejorar la inserción internacional de América Latina. Es decir, es el momento de actualizar la noción de "regionalismo abierto", reforzando la complementariedad entre los procesos de integración a la economía mundial y los esquemas de integración regional o subregional.

El punto obvio de partida es reconocer y aceptar las diferencias y las distintas visiones que han ido surgiendo, de modo de preservar el objetivo integracionista. No es realista ni plausible esperar que los países que han negociado TLC con los Estados Unidos o con la Unión Europea o con China los dejen caer o suponer que entre ellos no buscarán formas de mayor aproximación. El tema es exactamente como construir integración a partir de esas realidades diversas, buscando conciliarlas en aras de esquemas renovados de integración que eleven el atractivo de un mercado regional ampliado.

La integración puede y debe renovarse. Por un lado, consolidando y haciendo converger la red de acuerdos comerciales intra-regionales en materia de acceso, normas de origen y otros procedimientos y, por otro, actualizando sus compromisos en áreas no cubiertas, como servicios empresariales, servicios ambientales y compras públicas, por ejemplo. Ello es factible de realizar, preservando la idea central de un mercado ampliado, con libre movilidad de bienes y factores, avances serios hacia la coordinación macroeconómica, mecanismos de solución de controversias efectivamente vinculantes, un trato adecuado de las asimetrías, la gestación de fondos es-

\footnotetext{
${ }^{8} \mathrm{El}$ debate sobre los peligros de un "spaghetti bowl" de acuerdos comerciales preferenciales fue instalada por el profesor Jagdish Bhagwati y sus repercusiones trascienden el debate académico y tiene numerosas implicaciones prácticas, políticas y de economía política.
}

tructurales para conseguir beneficios equilibrados, coordinación en políticas sociales e iniciativas audaces en materia de energías renovables, infraestructura, economía verde y adaptación al cambio climático y al gran impulso ambiental.

La crisis de la integración es anterior a los TLC y es posible que tales negociaciones puedan colaborar en la puesta al día de la integración. Mientras en Centroamérica se estima que el Central America Free Trade Agreement (CAFTA por sus siglas en inglés) abre espacios para renovar y profundizar la integración y la Unión Aduanera, en América del Sur esa conclusión es menos compartida entre los diversos gobiernos. El tema sigue siendo cómo hacer para que estas negociaciones bilaterales no colisionen con la profundización de los esfuerzos de integración. Esto es un camino de doble vía ya que se requiere tanto disposición y flexibilidad de los países que cuentan con estos acuerdos bilaterales como de aquellos que no los tienen, en orden a explorar en conjunto vías que permitan elevar el atractivo y la pertinencia de la integración, con fórmulas realistas, graduales pero siempre enmarcadas en un proyecto estratégico de largo plazo.

Opciones para profundizar la integración hay muchas y variadas y probablemente la opción de un mercado común, con convergencia de políticas y con una institucionalidad comunitaria es la más exigente. La construcción gradual y realista de objetivos incrementales puede permitir avanzar en esa dirección pero el desafío actual debería ser bastante más modesto: preservar lo existente, cumplir los compromisos contraídos y tender puentes de convergencia entre las diversas agrupaciones subregionales.

\section{Desafíos del nuevo regionalismo y de la integración regional}

\section{La integración más allá del comercio}

Además de los beneficios asociados al libre comercio, la integración regional supone la gradual coordinación macroeconómica y en diversas políticas y normas más allá del campo comercial, incluyendo infraestructura, energía y ámbitos regulatorios, además de migración, previsión, salud, educación, medio ambiente, cambio climático, entre los más importantes. La experiencia europea incluye también políticas para reducir las asimetrías económicas entre sus miembros, para estimular la cohesión social en sus respectivas sociedades y para dotarse de una institucionalidad comunitaria que refleje el sentir balanceado del conjunto de sus integrantes. 


\section{El vínculo con China y Asia Pacífico}

Otro rasgo comercial novedoso es el creciente vínculo de economías de la región con Asia Pacífico. Este vínculo es más fuerte en América del Sur y se expresa fundamentalmente en la relación con China. Se trata de aprovechar el nuevo momento de las relaciones con China y con Asia Pacífico para avanzar en las tareas pendientes en integración subregional, generando condiciones de conectividad que, junto con reducir el costo de transporte de las exportaciones en dirección al Asia Pacífico, permitan también facilitar el intercambio y las inversiones intrarregionales.

\section{Regionalismo y comercio de servicios}

Una de las características de la nueva generación de acuerdos comerciales regionales es que muchos de ellos tienen como objetivo ir más allá de la liberalización del comercio de bienes e incluir compromisos para liberalizar el comercio de servicios. La pregunta que puede hacerse es si el comercio de servicios tiene alguna característica que lleve a una modificación o cambio en las conclusiones alcanzadas por el impacto de la liberalización del comercio preferencial en el caso de los bienes.

\section{Coordinación regulatoria: hacia una integración profunda}

Los gobiernos introducen normas para hacer frente a los problemas derivados de la falta de mercado, tales como la información asimétrica, las externalidades o los monopolios naturales. Dado que las barreras arancelarias se han reducido, las diferencias entre las legislaciones nacionales han actuado como barreras potencialmente importantes para el comercio. Si bien las diferencias entre las legislaciones nacionales pueden reflejar diferencias en las preferencias sociales, también existe el peligro de "captura del regulador" cuando se hace lobby por regulaciones a favor de los intereses del productor nacional, las cuales tienen un efecto de facto proteccionista. Paralelamente a los esfuerzos que se realizan en el sistema multilateral de comercio para desarrollar normas internacionales para reducir el impacto proteccionista de las normas, algunos acuerdos regionales de integración ahora persiguen una estrategia de regulación de coordinación para minimizar los impactos en el mercado de segmentación de las diferentes normativas nacionales.

\section{La aparición de "nuevos" desafíos a la integración}

Los nuevos desafíos habían estado bajo el velo del pensamiento económico neoclásico imperante en las últimas tres décadas del siglo pasado. Los nuevos desafíos, en verdad, no eran tan nuevos: involucraban aspectos señalados mucho antes por los pensadores latinoamericanos de mediados de siglo XX y reiteradamente por la CEPAL desde mediados de los años noventa. Estos desafíos estaban relacionados a la creación de las condiciones sobre las cuales basar el crecimiento económico y el desarrollo de la región: el perfeccionamiento de sistemas de interconexión de la infraestructura de transportes, de las telecomunicaciones, y del abastecimiento de energía en el subcontinente, el cual permitiese una mayor velocidad en los intercambios entre los países sudamericanos. A su vez, se hacía cada vez más visible la necesidad de plantear la construcción de coordinaciones productivas a nivel regional, a partir de la construcción de cadenas de valor regionales, que puedan competir a nivel internacional.

\section{La integración productiva en América Latina}

La integración productiva entre países es un concepto relativamente nuevo en el ámbito latinoamericano, aunque muy usado actualmente. López y Laplane (2004) relacionan el proceso de la integración productiva con el desarrollo de cadenas regionales de valor y complementariamente, con el análisis de la matriz productiva relevante para el cambio estructural. Reconocen el estatus creciente, entre los policy-makers, de las cadenas de valor en la explicación para la mejora de la competitividad de una región. La aceptación de esta visión, explican, se demuestra en la proliferación de iniciativas destinadas a fomentar estas cadenas en un gran número de países con diferentes tipos de desarrollo económico (Hernández et al. 2014åa 2014b). De una forma esquemática, los rasgos básicos que definen la integración productiva podrían describirse como la búsqueda de complementación entre las unidades productivas de diferentes zonas y países dentro de un bloque de integración regional, la especialización sectorial dentro de los bloques, y la búsqueda de una mayor competitividad a nivel región que permitan el avance. 

Integración productiva desde la óptica
de las economías pequeñas

Se propone avanzar en la investigación de la integración productiva desde la óptica de las economías pequeñas (Centroamérica y el Caribe). En primer lugar, se requiere la integración de la infraestructura, la cual permitiría una mayor interconexión entre las unidades productivas de los países, en las áreas estratégicas para el comercio actual: el transporte, la energía y las telecomunicaciones. En segundo lugar, se necesitan marcos normativos e iniciativas estatales para llevar adelante la competencia, complementación y especialización de los diversos actores productivos en diferentes cadenas de valor regionales, y redes de producción regional (Hernández, Hualde, Mulder y Sauvé 2016; Hernández et al $2014^{\mathrm{a}}$ y 2014 b). La complementación productiva de las unidades productivas siempre tiene en si elementos de cooperación y competencia que pueden ser resueltos por el mercado, pero que precisan de regulación estatal para florecer. En tercer lugar, la caída de las barreras arancelarias y no arancelarias que obstaculizan el proceso de integración en base a la dificultad de intercambiar bienes terminados, pero por sobre todas las cosas, los bienes intermedios, los cuales son la base para los intercambios intra-sectoriales que den lugar a redes de producción regional.

\section{Nuevo regionalismo abierto y produc- tividad}

Si se profundiza la integración con una óptica de regionalismo abierto, ésta podrá actuar como factor de productividad, al operar en mercados más abiertos a la competencia externa, complementando la inserción dinámica en los principales mercados internacionales y, al mismo tiempo, cerrando brechas productivas y sociales, induciendo el cambio estructural para la igualdad y favoreciendo una mayor presencia de las pymes en las corrientes de exportación.

\section{Instituciones, visiones nacionales y de largo plazo}

El mérito de los buenos esquemas de integración es que pueden pasar por encima de las diferencias entre los gobiernos que los componen pues se trata de apuestas de largo plazo, más allá de las coyunturas y de los gobiernos. Incorporar distintas visiones en un mismo marco institucional es lo que otorga solidez a las iniciativas de integración. De allí la necesidad de una institucionalidad media- dora que rompa los bloqueos políticos que puedan surgir entre los países miembros. Esta institucionalidad además debiera recoger la pluralidad de visiones nacionales y luego subregionales, de modo que sea esta pluralidad la que concurra a este esfuerzo de renovación de la integración regional (Rosales, 2008). El desafío consiste así en construir visiones de país, imágenes-objetivo y planificación de escenarios en materia de integración regional y nuevo regionalismo.

\section{La nueva arquitectura financiera re- gional}

La nueva arquitectura financiera regional es condición necesaria (aunque no suficiente) para impulsar el nuevo regionalismo y renovar los esfuerzos de una integración superior. Algunos de las propuestas de diseño de la Nueva Arquitectura Financiera que cambie la relación entre las finanzas y la producción se relacionan con la articulación entre el Estado, las corporaciones y la economía popular, y auspicie una renegociación en la división internacional del trabajo, se resaltan la atención a los problemas de los países en desarrollo, la reformulación de las Instituciones Financieras Internacionales y la transformación del sistema internacional de reservas sobre la base de arreglos bloque-regionales, entre otros.

\section{Referencias}

Alves Teixeira, R. y Desiderá Neto, W. A. (2012). La recuperación del desarrollismo en el regionalismo latino-americano. En Alves Teixeira, R. y Desiderá Neto, W. A., editores, Perspectivas para la integración de América Latina. IPEA, Brasilia.

Cárdenas, E., Ocampo, J. A., y Thorp, R. (2003). Industrialización y Estado en la América Latina: la leyenda negra de la posguerra. El Trimestre Económico. FCE.

Comisión Económica para América Latina y el Caribe (CEPAL) (1994). El regionalismo abierto en América Latina y el Caribe: la integración económica al servicio de la transformación productiva con equidad.

Comisión Económica para América Latina y el Caribe (CEPAL) (2016). Panorama de la Inserción Internacional de América Latina y el Caribe, 2016.

De la Mora Sánchez, L. M. (2018). Hacia dónde se dirige el régimen de comercio internacional y sus implicaciones para américa latina. 
Ethier, W. (1998). The New Regionalism. The Economic Journal, 108(449):1149-1161.

Hernández, R., Hualde, A., Muldery, N., y Sauvé, P. (2016). Innovation and Internationalization of Latin American Services (2016). Coedición CEPAL y Colegio de la Frontera Norte, Santiago.

Hettne, B. (1999). Globalization and the new regionalism: the second great transformation. En Globalism and the new regionalism, pp. 1-24. Springer.

Malamud, A. (2013). Overlapping Regionalism, No Integration: Conceptual Issues and the Latin American Experiences. European University Institute. Robert Schuman Centre for Advanced Studies Global Governance Programme. EUI Working Paper RSCAS 2013/20.

Robson, P. (1993). The new regionalism and developing countries. JCMS: Journal of Common Market Studies, 31(3):329-348.

Rosales, O. (2008). Integración regional: propuestas de renovación. En Altmann, J. y Rojas Aravena, F., editores, Las paradojas de la integración en América Latina y el Caribe, capítulo 3, pp. 3366. Siglo XXI; Fundación Carolina.

Rosenthal, G. (1998). Pensamiento y políticas sobre el desarrollo en América Latina y el Caribe: pasado y futuro. En Emmerij, L. y Núñez del Arco, J., editores, El desarrollo económico y social en los umbrales del siglo XXI, pp. 209-229. Banco Interamericano de Desarrollo, Washington D.C.

Rosenthal, G. (2004). ECLAC: a commitment to a Latin American way towards development, Unity and Diversity. En Berthelot, Y., editor, Development Ideas: Perspectives from the UN Regional Commissions. Indiana University Press, Bloomington.

Sanahuja, J. (2016). Regionalismo e integración en américa latina: de la fractura atlántico-pacífico a los retos de una globalización en crisis. Pensamiento propio, 21:29-76.

Schiff, M. y Winters, A. (2003). Regional Integration and Development. The World Bank, Washington D.C.

Söderbaum, F. y Shaw, T. M. (2003). Theories of new regionalism. Theories of new regionalism. New York: Palgrave Macmillan, pp. 1-21.

Viner, J. (1954). Schumpeter's history of economic analysis. The American Economic Review, 44(5):894-910.

Viner, J. (1999). The customs union issue. Trading Blocs: Alternative Approaches to Analyzing Preferential Trade Agreements, pp. 105-118. 